\title{
Exosomes: New Tuberculosis Biomarkers - Prospects From the Bench to the Clinic
}

\author{
Nicole A. Kruh-Garcia ${ }^{1}$, Jeff S. Schorey ${ }^{2}$ and Karen M. Dobos ${ }^{1}$ \\ ${ }^{1}$ Colorado State University \\ 2 University of Notre Dame \\ USA
}

\section{Introduction}

Biomarkers, derived from either the host (e.g. immunological markers, such as cytokines) or infectious agent (e.g. exported products, such as lipoarabinomannan), are indicative not only of disease but also of disease stage, severity and drug failure. Discovering new biomarkers from easily attainable bodily fluids is essential if we are to control tuberculosis, a diseases that kills 1.5 to 2 million individuals a year. The ideal biomarker could be used in the diagnosis and prognosis of disease from any suspect patient regardless of age, immune status or vaccination history. One potential biomarker source is exosomes; small membranebound vesicles released from cells which can be found circulating in the blood, and can be readily harvested for diagnostic testing. Exosomes shed from Mycobacterium tuberculosis $(M t b)$ infected samples (from in vitro produced to in vivo models and clinical samples) could provide an ideal reservoir of tuberculosis biomarkers. While it is possible to detect and monitor host and other bacterial components on exosomes, the scope of the following experiments are focused specifically on the $M t b$ proteins that are incorporated into exosomes.

\subsection{Detection of tuberculosis}

New diagnostic measures for the detection of tuberculosis are needed to identify and distinguish individuals with active tuberculosis from those harboring the dormant $M t b$ bacillus. The established method of TB detection for over seventy years is the tuberculin skin test (TST) or Mantoux test. This approach monitors the presence and diameter of induration as an indicator of the delayed-type hypersensitivity reaction to an intradermal injection of purified protein derivative (PPD) of $M t b$. Despite its worldwide use, there are several fundamental problems with the TST. First, while it can be used as an indicator of both the active disease and the asymptomatic, latent tuberculosis infection (LTBI), the test cannot differentiate between the two. Second, false positive results are frequent, most commonly due to a history of Bacille Calmette Guérin (BCG) vaccination. Similarly, due to the dominance of several conserved heat-shock protein in PPD, and their influence on the antigenicity of PPD (Yang, Troudt et al.), there is frequent cross-reactivity between infections with nontuberculosis mycobacterium (NTM) species (such as M. avium). Most 
disconcerting are false negative readings typically associated with impaired immune function (attributed to infections with HIV or with drug use).

The development of the interferon-gamma release assay (IGRA) has addressed some of the problems associated with the TST. Two types of IGRA test are currently commercially available, QuantiFERON-TB (Cellestis Limited) and T-SPOT-TB (Oxford Immunotec). Both measure the host biomarker, interferon-gamma (INF- $\gamma$ ) in whole-blood after stimulation with the mycobacterial antigens ESAT-6 and CFP10, by either ELISA or ELISpot assay, respectively. The T-SPOT-TB appears to be the most comprehensive diagnostic test to date (Table 1). Lastly, the Xpert MTB/RIF assay (Cephid) is a nucleic acid amplification based diagnostic and has the advantage of identifying resistance to rifampicin. However, since none of the aforementioned tests can distinguish between active and latent infections, sputum collection followed by microscopy (AFB smear) and culture, as well as chest radiography must be performed. The AFB smear test is simple, cost-effective and achieves high specificity; however this test suffers from a lack of sensitivity as on average it only detects $50 \%$ of active TB patients and even less in HIV positive patients and children.

\begin{tabular}{|c|c|c|c|c|c|c|}
\hline Test & $\begin{array}{c}\text { Detects } \\
\text { active } \\
\text { TB }\end{array}$ & $\begin{array}{c}\text { Detects } \\
\text { latent } \\
\text { TB }\end{array}$ & $\begin{array}{c}\text { Distinguishes } \\
\text { between } \\
\text { active and } \\
\text { latent TB }\end{array}$ & $\begin{array}{c}\text { Distinguishes } \\
\text { between BCG } \\
\text { vaccination } \\
\text { and } \text { Mtb } \\
\text { infection }\end{array}$ & $\begin{array}{c}\text { Distinguishes } \\
\text { between } \text { Mtb } \\
\text { and NTM } \\
\text { infection }\end{array}$ & $\begin{array}{c}\text { Reliable } \\
\text { results in } \\
\text { immune- } \\
\text { compromised } \\
\text { patients? }\end{array}$ \\
\hline AFB Smear & Yes & No & Yes & Yes & No & Yes $^{1}$ \\
\hline $\begin{array}{c}\text { AFB } \\
\text { culture }\end{array}$ & Yes & No & Yes & Yes & Yes & Yes $^{1}$ \\
\hline TST & Yes & Yes & No & No & No & No \\
\hline $\begin{array}{c}\text { Quanti- } \\
\text { FERON }\end{array}$ & Yes & Yes & No & Yes & ${\text { Yes }{ }^{2}}^{3}$ \\
\hline T-SPOT-TB & Yes & Yes & No & Yes & Yes $^{2}$ & Yes \\
\hline $\begin{array}{c}\text { Xpert } \\
\text { MTB/RIF }\end{array}$ & Yes & Yes & 3 & Yes & Yes & Yes \\
\hline $\begin{array}{c}\text { Clearview } \\
\text { TB ELISA }\end{array}$ & Yes & No & Yes & & No & Yes \\
\hline
\end{tabular}

${ }^{1}$ Provided that sputum can be obtained.

${ }^{2}$ This method can discriminate between most NTM (exceptions are M. gordonae, M. kansasii, M. szulgai and M. marinum); but it cannot distinguish between other members of the $M t b$ complex (M. bovis, $M$. africanum, M. microti and M. canetti).

${ }^{3}$ Data is limited.

Table 1. Comparison of a sampling of tuberculosis detection methods.

The ideal diagnostic would minimize false positives due to BCG vaccination and infections with NTM, eliminate false negatives associated with the immunocompromised and be able to distinguish between active and latent infections while permitting increased case detection via a robust, cheap, fast and sensitive assay for its use in high-burden, low-income countries. According to the World Health Organization (WHO), there were 9.4 million new cases of active tuberculosis (TB) with a prevalence of 14 million cases in 2009, which accounted for about 1.7 million deaths (WHO 2010). A serious issue is the inaccuracy of these estimations, 
with case detection rates (CDR) of only $63 \%$ (even lower in Africa) of pulmonary and other forms of the disease which is primarily due to undiagnosed and unreported cases (WHO 2010). Of those individuals newly infected with $M t b$, greater than one-tenth involved coinfection with HIV. Uncovering LTBI in at-risk individuals (e.g.. HIV positive, household contacts, those on immunosuppressive drugs) is critical so that the proper medical treatments can be administered to prevent future activation of the disease. Expeditious detection of smear negative cases, of which there were an estimated 2 million instances (WHO 2010), could potentially allow for treatment 4-6 weeks sooner.

\subsection{Identifying diagnostic biomarkers of TB}

There are two major classes of biomarkers - host response and pathogen generated. The majority of the research being conducted focuses on host biomarkers - including INF- $\gamma$ (as measured through the IGRA kits), other immunological markers (e.g.. cytokines, like IL-6 and TNF), host protein profiling, as well as, the production of antibodies to dominant $M t b$ antigens (such as, malate synthase and MPT51 (Wanchu, Dong et al. 2008)) (Walzl, Ronacher et al.). One of the primary concerns with using immune response as biomarkers of disease is the variation observed among the patient population due to numerous factors including secondary disease (causing increased/decreased inflammation) and altered response due to drug therapy. Alternatively, products made and released by $M t b$ during infection can also serve as biomarkers. Bacterial molecules including DNA (Cannas, Goletti et al. 2008) and lipoarabinomannan (LAM) (Minion, Leung et al.) can both be detected in urine during infection. Unlike most immunological biomarkers, bacterial products are specific to the tuberculosis. Moreover, identification and quantification of multiple $M t b$ products secreted/released during infection has the potential to be developed into a multiplex assay. Further, by monitoring changes in a panel of biomolecules, one could generate a fingerprint that could be used to indicate the different stages of disease (such as active versus latent).

To identify a combination of novel biomarkers for the generation of a point-of-care diagnostic test, we have begun screening human exosomes as a source of $M t b$ proteins. Preliminary data has proven that these vesicles are an untapped supply of pathogen derived biomarkers. There are several advantages to using exosomes: first, they can be isolated in a facile and expeditious manner. Second, they can be harvested from blood and urine - which is beneficial when sputum collection is not feasible (from children or in MDR cases). Third, our data indicates that $M t b$ proteins are not only contained, but also concentrated in these vesicles - allowing for greater sensitivity than can be achieved using unpurified whole blood or urine.

\subsection{Exosomes: Initial discoveries}

Exosomes were first described in mid-1980's by Johnstone et al. and Stahl et al. in reference to the small vesicles that bud from reticulocytes during maturation (Harding, Heuser et al. 1984; Johnstone, Adam et al. 1987). It was hypothesized that this process, was required to remove membrane-bound proteins such as the transferrin receptor from the maturing reticulocyte as these cells lack the lysosomes for protein degradation (Pan and Johnstone 1983). In a series of elegant electron microscopy studies, it was determined that the transferrin receptor (TR) was endocytosed and trafficked to the MVB where it was observed 
on $\sim 50 \mathrm{~nm}$ interluminal vesicles. MVBs containing the TR were shown to fuse with the plasma membrane and release the interluminal vesicles (Pan, Teng et al. 1985). These released vesicles were called exosomes.

Additional studies have shown that exosome release is not specific to maturing reticulocytes, as they are constitutively shed from most mammalian cells types including Bcells, macrophages and dendritic cells (Raposo, Nijman et al. 1996). Exosomes can be readily obtained from most bodily fluids, including but not limited to urine, plasma, breast milk, bronchoalveolar lavage (BAL) fluid and saliva (Admyre, Grunewald et al. 2003; Pisitkun, Shen et al. 2004; Caby, Lankar et al. 2005; Admyre, Johansson et al. 2007; Ogawa, KanaiAzuma et al. 2008) Exosomes released from antigen presenting cells contain MHC-I and MHC-II as well as CD86 and therefore are capable of antigen presentation (Zitvogel, Fernandez et al. 1999). Interestingly, exosomes derived from tumor cells have also been shown to induce an anti-tumor immune response, implicating a future utility in vaccine development (Zitvogel, Regnault et al. 1998).

\subsection{The advent of exosomes in tuberculosis research}

The $M t b$ cell wall is composed of a myriad of proteins and lipids, including the highly studied glycolipid, LAM. This molecule has been shown to be readily liberated from the mycobacteria during infection and has been correlated with several immunomodulatory functions (such as interactions with TLRs (Bhatnagar and Schorey 2007)). Beatty et al was the first to recognize LAM accumulation in MVBs and this report is consistent with subsequent exosomal release from infected cells (Beatty, Rhoades et al. 2000). Building upon initial evidence, Schorey et al characterized exosomes released from BCG-infected macrophages. Flow cytometry allowed for the identification of vesicles positive for MHC-II, Hsp70, and LAMP1, as well as mycobacterial pathogen-associated molecular patterns (PAMPs) (Bhatnagar, Shinagawa et al. 2007; Giri and Schorey 2008). They went on to demonstrate the exosomes released from mycobacterial-infected macrophages could activate both the inate and acquired immune responses in vitro and in vivo (Blood, 2007 and PLoS One 2008). In addition, it was shown that exosomes isolated from the BAL fluid of $M$. bovis BCG infected mice contained LAM and the 19kDa antigen (Bhatnagar, Shinagawa et al. 2007).

\subsection{Concentration and siphoning of biomarkers in exosomes}

Exosome biology is a relatively new field with roughly 1000 primary journal articles and over 200 reviews (Raimondo, Morosi et al. 2011). Exosomes have been studied with techniques such as western blotting, microscopy, FACS and mass spectrometry. Characterization of exosomal contents, resulted in the identification of a number of host, as well as pathogen derived products, including: lipids (Laulagnier, Motta et al. 2004) and nucleic acids (miRNA and mRNA) (Valadi, Ekstrom et al. 2007), in addition to proteins. However, only a few of these studies utilize proteomics to decipher the contents of these vesicles and their relationship to various diseases, such as cancers, diabetes, prenatal disorders and renal diseases (Ben Ameur, Molina et al. 2010; Diaz, Pinto et al. 2011; Moon, You et al. 2011). Proteomics was performed on purified exosomes as early as 2004 (Bard, Hegmans et al. 2004). Defining the proteomic content of exosomes derived from urine and 
blood has been key to biomarker discovery in several diseases (Bard, Hegmans et al. 2004; Mears, Craven et al. 2004; Thomas, Sexton et al. 2010). However, the mining of exosomederived proteins related to infectious diseases has yet to be exploited. The discovery that mycobacterial products could be found in exosomes release from infected animals led to the first full-scale attempt to characterize the $M t b$ exo-proteome (comprehensive collection of proteins capable of being secreted from $M t b$-infected cells within exosomes) (Giri, Kruh et al. 2010). The objective of this research is to fuel biomarker discovery utilizing mass spectrometry based approaches.

\section{The Mtb exo-proteome: in vitro}

Based on the hypothesis that exosomes are a novel source of bacterial components which could be exploited for biomarker identification, we set out to define the mycobacterial proteins contained in exosomes released from $M t b$-infected macrophages. Exosomes were purified from $M t b$ infected macrophages using differential centrifugation and sucrose gradient (Griffiths, Heesom et al. 2007; Giri, Kruh et al. 2010). The exosomes contained the characteristic size and shape as defined by EM (Figure 1).
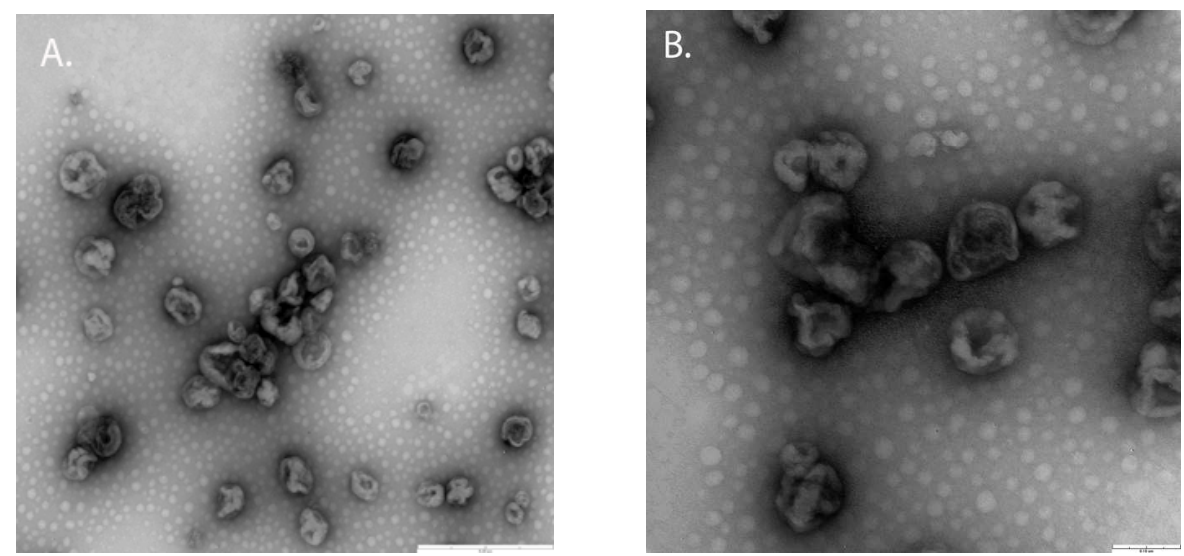

Fig. 1. Electron micrograph of exosomes purified from $M t b$ infected J774a.1 cells at (A) 3000x (bar $=0.5 \mu \mathrm{m}$ ) and $(\mathrm{B}) 8000 \mathrm{x}$ magnification $(\mathrm{bar}=0.1 \mu \mathrm{m})$. The vesicles showed the size $(30$ $100 \mathrm{~nm}$ ) and spherical or cup-like shape expected of exosomes.

\subsection{Proteomics of exosomes released from Mtb-infected macrophages}

Consistent with previous publications (Bhatnagar, Shinagawa et al. 2007), the host exosomal marker LAMP-1, as well as mycobacterial LAM and $19 \mathrm{kDa}$ antigen (Rv3763) were detected via western blot (data not shown). Several known secreted proteins previously undetected in exosomes, including KatG (Rv1908c), the Ag85 complex (Rv3804c, Rv1886c and Rv0129c), GroES (Rv3418c) and CFP10 (Rv3874), were also demonstrated by western blot analysis (Giri, Kruh et al. 2010). In addition to these proteins, we identified roughly forty mycobacterial proteins by MS, of which $95 \%$ were previously defined either experimentally or through predictive algorithms to be secreted (Table 2) (Rosenkrands, King et al. 2000; Malen, Berven et al. 2007; Giri, Kruh et al. 2010). One can hypothesize that the mycobacterial 
proteins released into the phagosome or cytoplasm are transported to an MVB, incorporated into the interluminal vesicles and released from the infected cell via exosomes into various bodily fluids (Figure 2). As indicated earlier, the exosomes containing both host and mycobacterial components can modulate the host immune response. Similar experiments were performed with irradiated (dead) $M t b$ and only 1 mycobacterial protein was identified. Whether this difference between live and dead $M t b$ was due to differential transport of the mycobacteria within the macrophage or a requirement for mycobacterial metabolic activity requires further investigation.

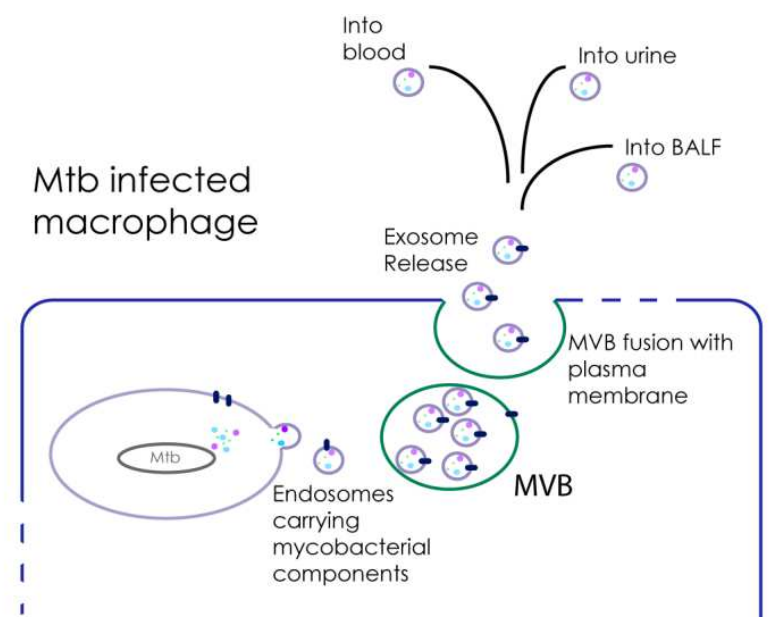

Fig. 2. Hypothesized pathway of exosome secretion in $M t b$-infected cells. Based on experimental evidence, antigenic proteins are secreted within the $M t b$-containing host compartment, packaged and shuttled into multivesicular bodies. The MVBs can fuse with the plasma membrane to release the exosomes into the peripheral fluids.

In addition, J774a.1 macrophages were exposed to culture filtrate proteins (CFP), a complex mixture of over 200 proteins secreted from $M t b$ during in vitro growth (Sonnenberg and Belisle 1997). Exosomes released during this experiment were collected and analyzed after in-gel digestion. The mycobacterial proteins identified from the CFP-treated exosomes included many well-characterized immunedominant antigens and were remarkably similar $(>85 \%)$ to those isolated from J774a.1 cells infected with live $M t b$. As previously mentioned, exosomes are being evaluated as tumor vaccines and the presence of highly antigenic mycobacterial proteins on exosomes from CFP-treated macrophages suggests that they too may be a viable vaccine candidates for TB. This hypothesis is presently under investigtion.

These aforementioned in vitro experiments afforded us with enough exosomal material to build our methodology and lay the foundation for the analysis of exosomes isolated from $M t b$-infected mice, where samples more limited. In prior studies of exosomes, mycobacterial proteins were identified only by western blot. However, mass spectrometry allows for the identification of proteins without the use of antibodies (which are only available to a small subset of proteins). Moreover, while $\sim 8 \mu \mathrm{g}$ of exosomes is required for each western blot (identifying only one protein per blot), a parallel sample subject to MS analysis, allows for the discrimination of hundreds of proteins of both host and bacterial origin. 


\begin{tabular}{|lcc|}
\hline Rv \# & Protein Name & $\begin{array}{c}\text { Relative } \\
\text { Abundance (\%) }\end{array}$ \\
\hline Rv2220 & GlnA1 & 13.05 \\
Rv1860 & Mpt32/APA & 12.34 \\
Rv1886c & Antigen 85-B & 9.58 \\
Rv3804c & Antigen 85-A & 8.92 \\
Rv1980c & Mpt64 & 6.89 \\
Rv1908c & KatG & 4.43 \\
Rv3418c & GroES & 3.59 \\
Rv0934 & PstS1 & 3.41 \\
Rv3248c & SahH & 3.17 \\
Rv0462 & LpdC & 2.87 \\
Rv1926c & Mpt63 & 2.46 \\
Rv1355c & MoeY & 2.22 \\
Rv2244 & AcpM & 2.16 \\
Rv2031c & HspX & 1.86 \\
Rv1932 & Cfp20 & 1.80 \\
\hline
\end{tabular}

Table 2. Sampling of proteins identified in exosomes from H37Rv-infected J774a.1 cells by LC-MS-MS. See Giri, Kruh et al. 2010, for the extended list. The fifteen most abundant mycobacterial proteins are listed, based on the total number of spectral counts from both insolution and in-gel processing (compiled in the aforementioned paper plus additional unpublished experiments). Relative abundance was calculated using the following formula: (total \# of spectral counts for a given protein/cumulative total \# of mycobacterial spectral counts) ${ }^{*} 100$.

The primary analysis from in vitro derived exosomes, provides an extended candidate list for the study of $M t b$ biomarkers. Our next step in the biomarker discovery pipeline is to verify that these results translate to an in vivo system.

\section{The Mtb exo-proteome: in vivo}

Since tuberculosis is primarily a respiratory affliction, the initial in vivo investigation was performed utilizing exosomes isolated from BAL fluid to determine if mycobacterial proteins could be identified from an infected animal model. The hypothesis is that the protein composition of BAL fluid exosomes will reflect the initial in vitro findings and the proteins identified will change over the course of the infection. This may provide the starting point for making correlations between exosomal content and stage of infection. The end goal is find "peptide fingerprints" which represent the mycobacterial proteins most commonly present in exosomes of patients with active or latent tuberculosis.

\subsection{Proteomics of exosomes released into the bronchoalveolar lavage fluid of $M$ tb- infected mice}

BAL fluid was collected from mice infected via aerosol with either wild-type (H37Rv) or a relA deficient mutant of $M t b$. Both the concentration and $M t b$ protein content of the exosomes were monitored over time. To accomplish this, we employed similar MS methodology as in our in vitro analysis (Giri, Kruh et al. 2010). MS analysis of BAL fluid 
derived exosomes from $\mathrm{H} 37 \mathrm{Rv} M t b$-infected mice over the different time points revealed a significant overlap (50\%) with the mycobacterial proteins previously identified in the in vitro tissue culture experiments (Figure 3). No mycobacterial proteins were identified in exosomes purified from BAL fluid on day 1 post-infection or as expected, in naïve mice. In all other time points, the number of mycobacterial spectral counts (data not shown) as well as the proteins identified changed over time (Figure 4). In exosomes from both H37Rv and the relA mutant infections, the largest number of proteins were identified early in the infection (day 14), however the number of proteins identified from the relA mutant was half that of the wt strain. Interestingly, during the wt infection, the exosomes that yielded the highest total mycobacterial spectral count was at day 56. Moreover, the exosomes from wtinfected mice showed on average three times as many spectral counts per time point than the relA mutant (data not shown). This is likely, at least in part, due to the differences in bacterial load between wt and $\Delta$ RelA-infected mice (10 to 100 fold difference in lung colony forming units at different time points). In both wt and $\Delta$ RelA-infected mice there were some proteins present on exosomes which were there throughout the course of disease, while others were transiently expressed.

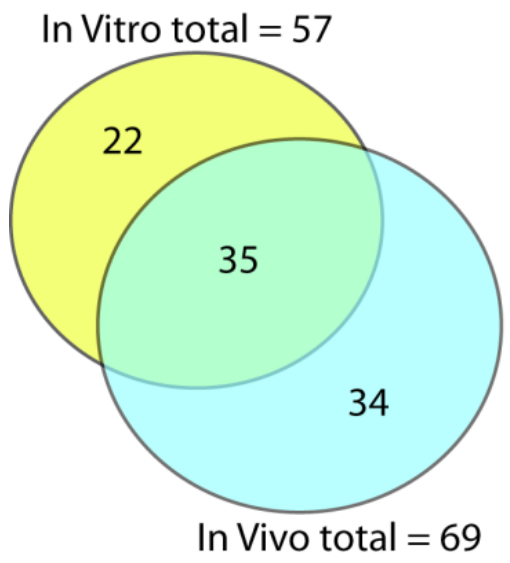

Fig. 3. Venn diagram depicting the number of mycobacterial proteins identified in exosomes from H37Rv-infected macrophages (yellow), the BAL fluid of H37Rv-infected mice (blue), and both (overlap). 
A.

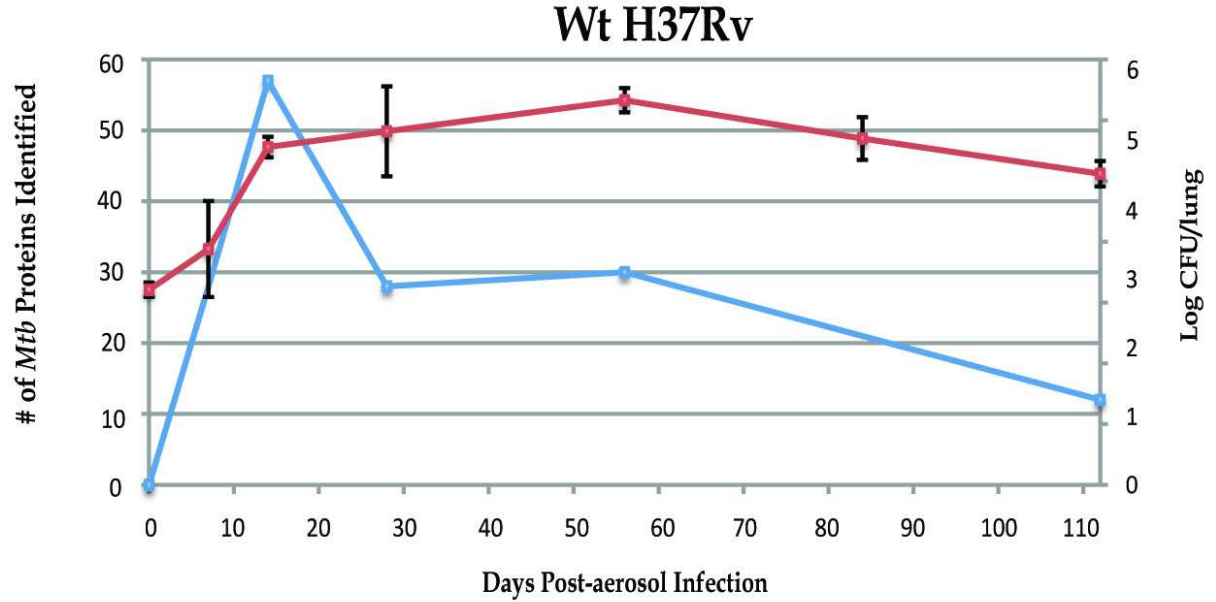

B.

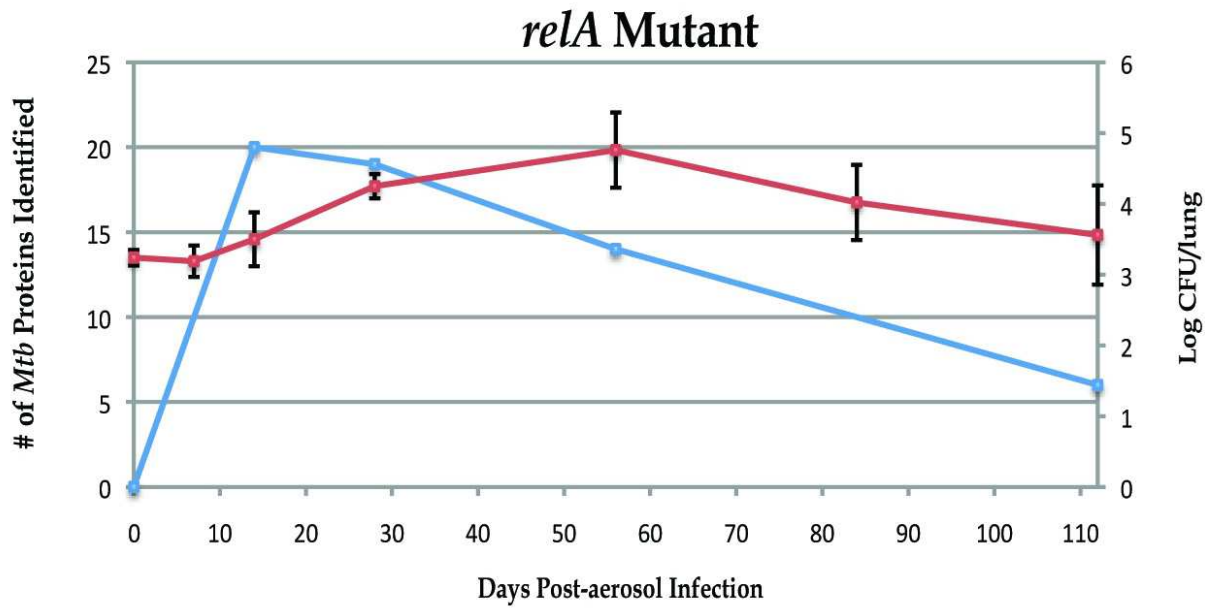

Fig. 4. CFUs versus $M t b$ proteins identified over time in (A) H37Rv $M t b$-infected mice and (B) $\Delta$ RelA-infected mice. The red lines indicate the Log CFU in the lung over time, as indicated on the right $y$-axius. CFUs are representative of the bacterial load of the lungs of 5 mice per time point. The ble line represents the number of proteins identified by MS over the course of the infection, as measured on the left y-axis. 
While majority of the 15 most dominant mycobacterial proteins found in exosomes released from infected J774a.1 cells were seen in the mouse exosomes (Table 3), many of them were less abundant (e.g.. KatG, SahH and Cfp20) and several of these proteins were absent entirely (e.g. LpdC and MoeY). Most interesting are several striking differences in the mycobacterial protein identified on exosomes from BALfluid when mice were infected with wt or relA mutant. The relA gene is annotated as being a non-essential gene, based on viability of the strain subsequent to the insertion of the Himar1 transposon (Lamichhane, Zignol et al. 2003). Biochemical characterization of the RelA protein suggests that this enzyme has both 3'-pyrophosphoryltransferase and pyrophosphohydrolase activities. Functional deletion of this protein results in the depletion of hyperphophorylated guanosine ((p)ppGpp), which can decrease protein synthesis leading to effects on long term growth and the ability to transition in and out of dormancy (Avarbock, Salem et al. 1999; Primm, Andersen et al. 2000; Klinkenberg, Lee et al. 2010). Dahl et al. has reported that the gene expression level of several secreted proteins (including the Antigen 85 complex and GroES) are increased in the relA mutant (Dahl, Kraus et al. 2003), however, based on spectral counts, the majority of the secreted proteins identified in exosomes display the opposite trend (Figure 5). While a more quantitative approach is necessary to truly hone in on the differences between the wt and $\mathrm{rel} A$ mutant, this preliminary data suggest that there is a qualitative and quantitative difference in proteins targeted to MVB/exosomes when macrophages are infected with wt compared to relA mutant in vivo. Future experiments, including multiple reaction monitoring (MRM)-MS analysis will allow for the monitoring of the intensity of specific peptides in each sample, while allowing one to ignore the $\mathrm{m} / \mathrm{z}^{\prime} \mathrm{s}$ corresponding to host peptides.

\begin{tabular}{|llcccc|}
\hline \multirow{2}{*}{ Rv \# } & \multirow{2}{*}{ Protein Name } & $\begin{array}{c}\text { Day } \\
\mathbf{1 4}\end{array}$ & $\begin{array}{c}\text { Day } \\
\mathbf{2 8}\end{array}$ & $\begin{array}{c}\text { Day } \\
\mathbf{5 6}\end{array}$ & $\begin{array}{c}\text { Day } \\
\mathbf{1 1 2}\end{array}$ \\
\hline Rv2220 & GlnA1 & + & $*$ & $*$ & $*$ \\
Rv1860 & Mpt32/APA & + & $*$ & $*$ & $\Delta$ \\
Rv1886c & Antigen 85-B & + & + & + & + \\
Rv3804c & Antigen 85-A & + & + & + & + \\
Rv1980c & Mpt64 & + & + & + & $*$ \\
Rv1908c & KatG & $*$ & $*$ & $*$ & - \\
Rv3418c & GroES & + & + & $*$ & $*$ \\
Rv0934 & PstS1 & + & + & $*$ & - \\
Rv3248c & SahH & $*$ & + & $*$ & - \\
Rv0462 & LpdC & - & - & - & - \\
Rv1926c & Mpt63 & $*$ & + & $*$ & - \\
Rv1355c & MoeY & - & - & - & - \\
Rv2244 & AcpM & + & + & + & $\Delta$ \\
Rv2031c & HspX & + & + & + & $*$ \\
Rv1932 & Cfp20 & $*$ & $*$ & $*$ & - \\
\hline
\end{tabular}

Table 3. Comparison of the fifteen most abundant proteins originally identified in exosomes from H37Rv-infected J774a.1 cells (Table 2) to their presence $(+)$ or absence (-) in BAL fluid exosomes at various time-points during the infection of Balc/c mice. $+=$ protein detected in both H37Rv and relA mutant exosomes; * = presence in H37Rv exosomes only; $\Delta=$ only in relA mutant exosomes. 


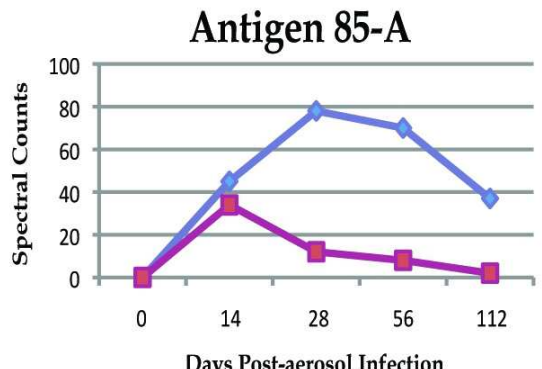

Days Post-aerosol Infection

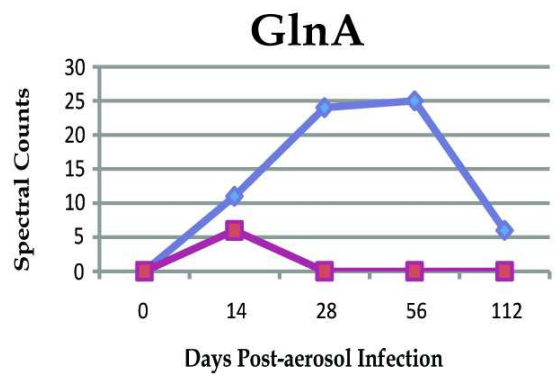

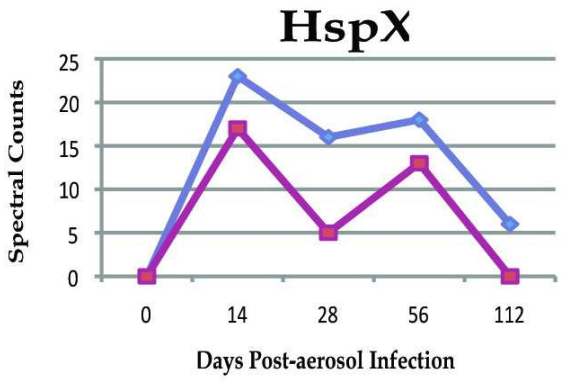

Mpt63

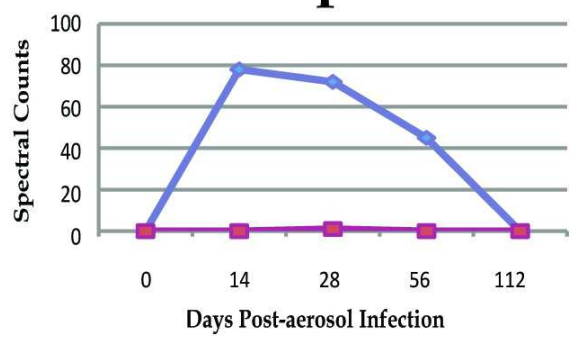

Fig. 5. Kinetics of various proteins during the course of the wild-type H37Rv (blue) compared to the relA mutant (red) infection, including GlnA, Antigen 85-A, HspX and Mpt63. These graphs emphasize the range of spectral counts and the pattern of individual protein expression. Transposon mutants effecting the expression of most of these proteins have led to the hypothesis that many of these proteins (HspX, Ag-85a, Mpt63) are "nonessential" (Sassetti, Boyd et al. 2003) - but their presence in exosomes is an indicator that their expression may be triggered during infection $(\mathrm{HspX}$, as seen by transcipts $(\mathrm{Hu}$, Mangan et al. 2000; Dubnau, Fontan et al. 2002) and not in a simple culture.

\section{Conclusions: Future of biomarkers in tuberculosis}

Tuberculosis research in diagnostics has suffered from a lack of resources for the discovery and down-stream development of a cheap and facile clinical assay for the detection of active $\mathrm{TB}$ and to distinguish active from latent infection. This is due in part to the reliance on the TST; which for decades provided a cheap assay for detection of Mtb infection, and by our reliance on sputum smear tests to detect active disease. Most would argue that neither are optimal means to detect and diagnose TB nor evaluate drug treatment. However, in the absence of assays that could replace these tests without increases in cost, these remain the gold standard. The $M t b$ proteome has been mined for decades in pursuit of a serodiagnostic or celluar assay for tuberculosis, and the development and use of IGRA tests represent a hallmark of success. The outlook for a serodiagnostic assay appears more dismal, due to the requirement of either a predictable antibody response or high bacterial product shedding into the blood or BAL fluid. Our initial identification of $M t b$ proteins in BAL fluid exosomes 
was a stepping-stone in the process of novel discovery of resources for development of second-generation diagnostic and prognostic assays. While BAL fluid is not the most ideal fluid to collect from patients, its isolation from mice and the use of it in the discovery phase of biomarker identification is suitable if these $M t b$ exo-proteome findings translate to other bodily fluids. Exosomes can be isolated from urine and serum, both of which are easier to collect than sputum. Harvesting and characterization of sera and urine exosomes are commonplace in diseases such as cancer. Consistent with our findings in the mouse model, our preliminary evidence from a panel of five TB patients and five household contacts suggests that sera analyzed from patients with active disease contain on average over 3000 $\mu \mathrm{g}$ of exosomes per $\mathrm{mL}$, which is over 30 times greater than the concentration of exosomes in the control sera. This makes exosomes an attractive target for pathogen-associated diagnostic biomarkers. Since many diagnostics are unreliable in immune compromised individuals, the allure of an exosome-based diagnostic will be enhanced if the exosomes undergo a similar rise in individuals co-infected with HIV.

Bodily fluids such as urine and blood are highly complex (with an estimate of $>10^{6}$ protein products in human plasma (Anderson and Hunter 2006)). The purification of exosomes from these samples reduces the amount of biocomponents resulting in a drastic enrichment. Numerous attempts have been made using MS to search for mycobacterial proteins in whole blood or urine. Unfortunately the results from these studies were not encouraging as even immunodepletion (with a MARS column) and various fractionation techniques (e.g. SCX and $\mathrm{mRP}$ on an HPLC), did not lead to the identification of a significant number of mycobacterial proteins in blood or urine. One major benefit to isolating exosomes from these fluids prior to proteomic studies is that these vesicles not only serve as a mechanism of concentrating the mycobacterial proteins, but they are also an effective method of removing major contaminants found in bodily fluids.

Urine is an established, readily attainable source of exosomes (Pisitkun, Shen et al. 2004). As previously mentioned, LAM (and mycobacterial DNA) has been found in urine of $M t b$ infected patients, including those with HIV. One kit currently on the market for the detection of LAM in urine is the Clearview TB ELISA (Inverness Medical Innovations Inc.). How LAM is secreted into the urine is questionable, however one can hypothesize that transport from the site of infection into the urine can occur through exosomes - either by encapsulation or incorporation into the lipid membrane of the vesicle. It is possible that LAM could be an additional marker in combination with $M t b$ proteins in a future clinical assay. Although at this point, like all proteins identified in exosomes, the concentration of LAM per volume of exosome would need to be quantified at various time-points during infection.

The discovery phase of identifying biomarkers from exosomes is in its infancy. While we have the potential to study fluctuations in exosomal host proteins too, deciphering these differences can be tricky due to additional changes in immune response and reactions to secondary infections or inflammatory disease. In the end, the best diagnostic may involve monitoring numerous proteins, perhaps both host and pathogen derived. The content of exosomes has the potential not only for use in diagnostics, but also additional predictive value in the classification of disease status, assessment of therapeutics and overall prognosis. 
The use of quantitative proteomics and MRM technology will be key in narrowing down the list of candidates. While this method is not necessarily suitable for the clinical lab (especially those in resource-limited areas) or is it cost effective - it is a mechanism to define biomarkers that can be implemented in a more cost effective diagnostic tool, such as an ELISA, or microfluidic "dip-test" that could be performed in a resource poor setting.

Another important consideration is the method used for exosome purification. At present the gold standard calls for differential centrifugation followed by sucrose gradient. Although this results is highly purified exosomes it is not suitable for high throughput analysis of serum/urine samples and cannot be used in the setting where TB diagnostics is most needed. Recently there have been a number of new purification techniques that have been developed include microfiltration using low protein-binding size exclusion filters, sizeexclusion chromatography, and microfluidics. ExoQuick ${ }^{\mathrm{TM}}$, a recently developed commercial product from System Biosciences, allows for rapid isolation of exosomes based on a simple precipitation process and shows promise as a purification technique amenable to a point-of-care diagnostics.

\section{Acknowledgements}

This work was supported through grants AI056979 and AI052439 (J. S. S.) and HHSN266200400091c (K.M.D) from the National Institute of Allergy and Infectious Diseases (NIAID). We would like to thank Pramod Giri for isolation of exosomes from mouse BAL fluid and EM photos. The antibodies to the various mycobacterial proteins as well as the $M t b$ culture filtrate proteins were provided from Colorado State University as part of NIH, NIAID Contract No. HHSN266200400091C, entitled "Tuberculosis Vaccine Testing and Research Materials". Mouse infections and BAL fluid collection were performed under William Bishai's laboratory at Johns Hopkins School of Medicine and funded under the "Tuberculosis Animal Research and Gene Evaluation Taskforce", NIH/NIAID N01AI30036. The authors graciously thank Lisa M. Wolfe for critique of this manuscript.

\section{References}

Admyre, C., J. Grunewald, et al. (2003). "Exosomes with major histocompatibility complex class II and co-stimulatory molecules are present in human BAL fluid." Eur Respir J 22(4): 578-583.

Admyre, C., S. M. Johansson, et al. (2007). "Exosomes with immune modulatory features are present in human breast milk." J Immunol 179(3): 1969-1978.

Anderson, L. and C. L. Hunter (2006). "Quantitative mass spectrometric multiple reaction monitoring assays for major plasma proteins." Mol Cell Proteomics 5(4): 573-588.

Avarbock, D., J. Salem, et al. (1999). "Cloning and characterization of a bifunctional RelA/SpoT homologue from Mycobacterium tuberculosis." Gene 233(1-2): 261269.

Bard, M. P., J. P. Hegmans, et al. (2004). "Proteomic analysis of exosomes isolated from human malignant pleural effusions." Am J Respir Cell Mol Biol 31(1): 114-121. 
Beatty, W. L., E. R. Rhoades, et al. (2000). "Trafficking and release of mycobacterial lipids from infected macrophages." Traffic 1(3): 235-247.

Ben Ameur, R., L. Molina, et al. (2010). "Proteomic approaches for discovering biomarkers of diabetic nephropathy." Nephrol Dial Transplant 25(9): 2866-2875.

Bhatnagar, S. and J. S. Schorey (2007). "Exosomes released from infected macrophages contain Mycobacterium avium glycopeptidolipids and are proinflammatory." J Biol Chem 282(35): 25779-25789.

Bhatnagar, S., K. Shinagawa, et al. (2007). "Exosomes released from macrophages infected with intracellular pathogens stimulate a proinflammatory response in vitro and in vivo." Blood 110(9): 3234-3244.

Caby, M. P., D. Lankar, et al. (2005). "Exosomal-like vesicles are present in human blood plasma." Int Immunol 17(7): 879-887.

Cannas, A., D. Goletti, et al. (2008). "Mycobacterium tuberculosis DNA detection in soluble fraction of urine from pulmonary tuberculosis patients." Int J Tuberc Lung Dis 12(2): 146-151.

Dahl, J. L., C. N. Kraus, et al. (2003). "The role of RelMtb-mediated adaptation to stationary phase in long-term persistence of Mycobacterium tuberculosis in mice." Proc Natl Acad Sci U S A 100(17): 10026-10031.

Diaz, S. O., J. Pinto, et al. (2011). "Metabolic biomarkers of prenatal disorders: an exploratory NMR metabonomics study of 2nd trimester maternal urine and blood plasma." J Proteome Res.

Dubnau, E., P. Fontan, et al. (2002). "Mycobacterium tuberculosis genes induced during infection of human macrophages." Infect Immun 70(6): 2787-2795.

Giri, P. K., N. A. Kruh, et al. (2010). "Proteomic analysis identifies highly antigenic proteins in exosomes from M. tuberculosis-infected and culture filtrate protein-treated macrophages." Proteomics 10(17): 3190-3202.

Giri, P. K. and J. S. Schorey (2008). "Exosomes derived from M. Bovis BCG infected macrophages activate antigen-specific CD4+ and CD8+ T cells in vitro and in vivo." PLoS One 3(6): e2461.

Griffiths, R. E., K. J. Heesom, et al. (2007). "Normal prion protein trafficking in cultured human erythroblasts." Blood 110(13): 4518-4525.

Harding, C., J. Heuser, et al. (1984). "Endocytosis and intracellular processing of transferrin and colloidal gold-transferrin in rat reticulocytes: demonstration of a pathway for receptor shedding." Eur J Cell Biol 35(2): 256-263.

$\mathrm{Hu}$, Y., J. A. Mangan, et al. (2000). "Detection of mRNA transcripts and active transcription in persistent Mycobacterium tuberculosis induced by exposure to rifampin or pyrazinamide." J Bacteriol 182(22): 6358-6365.

Johnstone, R. M., M. Adam, et al. (1987). "Vesicle formation during reticulocyte maturation. Association of plasma membrane activities with released vesicles (exosomes)." J Biol Chem 262(19): 9412-9420.

Klinkenberg, L. G., J. H. Lee, et al. (2010). "The stringent response is required for full virulence of Mycobacterium tuberculosis in guinea pigs." J Infect Dis 202(9): 13971404. 
Lamichhane, G., M. Zignol, et al. (2003). "A postgenomic method for predicting essential genes at subsaturation levels of mutagenesis: application to Mycobacterium tuberculosis." Proc Natl Acad Sci U S A 100(12): 7213-7218.

Laulagnier, K., C. Motta, et al. (2004). "Mast cell- and dendritic cell-derived exosomes display a specific lipid composition and an unusual membrane organization." Biochem J 380(Pt 1): 161-171.

Malen, H., F. S. Berven, et al. (2007). "Comprehensive analysis of exported proteins from Mycobacterium tuberculosis H37Rv." Proteomics 7(10): 1702-1718.

Mears, R., R. A. Craven, et al. (2004). "Proteomic analysis of melanoma-derived exosomes by two-dimensional polyacrylamide gel electrophoresis and mass spectrometry." Proteomics 4(12): 4019-4031.

Minion, J., E. Leung, et al. "Diagnosing tuberculosis with urine lipoarabinomannan: systematic review and meta-analysis." Eur Respir J.

Moon, P. G., S. You, et al. (2011). "Urinary exosomes and proteomics." Mass Spectrom Rev.

Ogawa, Y., M. Kanai-Azuma, et al. (2008). "Exosome-like vesicles with dipeptidyl peptidase IV in human saliva." Biol Pharm Bull 31(6): 1059-1062.

Pan, B. T. and R. M. Johnstone (1983). "Fate of the transferrin receptor during maturation of sheep reticulocytes in vitro: selective externalization of the receptor." Cell 33(3): 967-978.

Pan, B. T., K. Teng, et al. (1985). "Electron microscopic evidence for externalization of the transferrin receptor in vesicular form in sheep reticulocytes." J Cell Biol 101(3): 942948.

Pisitkun, T., R. F. Shen, et al. (2004). "Identification and proteomic profiling of exosomes in human urine." Proc Natl Acad Sci U S A 101(36): 13368-13373.

Primm, T. P., S. J. Andersen, et al. (2000). "The stringent response of Mycobacterium tuberculosis is required for long-term survival." J Bacteriol 182(17): 4889-4898.

Raimondo, F., L. Morosi, et al. (2011). "Advances in membranous vesicle and exosome proteomics improving biological understanding and biomarker discovery." Proteomics 11(4): 709-720.

Raposo, G., H. W. Nijman, et al. (1996). "B lymphocytes secrete antigen-presenting vesicles." J Exp Med 183(3): 1161-1172.

Rosenkrands, I., A. King, et al. (2000). "Towards the proteome of Mycobacterium tuberculosis." Electrophoresis 21(17): 3740-3756.

Sassetti, C. M., D. H. Boyd, et al. (2003). "Genes required for mycobacterial growth defined by high density mutagenesis." Mol Microbiol 48(1): 77-84.

Thomas, C. E., W. Sexton, et al. (2010). "Urine collection and processing for protein biomarker discovery and quantification." Cancer Epidemiol Biomarkers Prev 19(4): 953-959.

Valadi, H., K. Ekstrom, et al. (2007). "Exosome-mediated transfer of mRNAs and microRNAs is a novel mechanism of genetic exchange between cells." Nat Cell Biol 9(6): 654-659.

Walzl, G., K. Ronacher, et al. "Immunological biomarkers of tuberculosis." Nat Rev Immunol 11(5): 343-354. 
Wanchu, A., Y. Dong, et al. (2008). "Biomarkers for clinical and incipient tuberculosis: performance in a TB-endemic country." PLoS One 3(4): e2071.

WHO (2010). Global Tuberculosis Report, World Health Organization.

Yang, H., J. Troudt, et al. "Three protein cocktails mediate delayed-type hypersensitivity responses indistinguishable from that elicited by purified protein derivative in the guinea pig model of Mycobacterium tuberculosis infection." Infect Immun 79(2): 716-723.

Zitvogel, L., N. Fernandez, et al. (1999). "Dendritic cells or their exosomes are effective biotherapies of cancer." Eur J Cancer 35 Suppl 3: S36-38.

Zitvogel, L., A. Regnault, et al. (1998). "Eradication of established murine tumors using a novel cell-free vaccine: dendritic cell-derived exosomes." Nat Med 4(5): 594600 . 


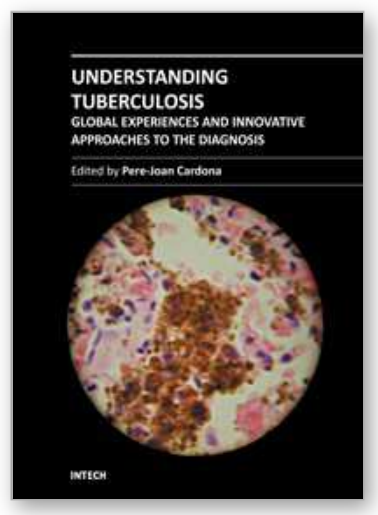

\section{Understanding Tuberculosis - Global Experiences and Innovative Approaches to the Diagnosis}

Edited by Dr. Pere-Joan Cardona

ISBN 978-953-307-938-7

Hard cover, 552 pages

Publisher InTech

Published online 15, February, 2012

Published in print edition February, 2012

Mycobacterium tuberculosis is a disease that is transmitted through aerosol. This is the reason why it is estimated that a third of humankind is already infected by Mycobacterium tuberculosis. The vast majority of the infected do not know about their status. Mycobacterium tuberculosis is a silent pathogen, causing no symptomatology at all during the infection. In addition, infected people cannot cause further infections. Unfortunately, an estimated 10 per cent of the infected population has the probability to develop the disease, making it very difficult to eradicate. Once in this stage, the bacilli can be transmitted to other persons and the development of clinical symptoms is very progressive. Therefore the diagnosis, especially the discrimination between infection and disease, is a real challenge. In this book, we present the experience of worldwide specialists on the diagnosis, along with its lights and shadows.

\section{How to reference}

In order to correctly reference this scholarly work, feel free to copy and paste the following:

Nicole A. Kruh-Garcia, Jeff S. Schorey and Karen M. Dobos (2012). Exosomes: New Tuberculosis Biomarkers - Prospects From the Bench to the Clinic, Understanding Tuberculosis - Global Experiences and Innovative Approaches to the Diagnosis, Dr. Pere-Joan Cardona (Ed.), ISBN: 978-953-307-938-7, InTech, Available from: http://www.intechopen.com/books/understanding-tuberculosis-global-experiences-and-innovative-approachesto-the-diagnosis/tuberculosis-biomarkers-prospects-from-the-bench-to-the-clinic

\section{INTECH}

open science | open minds

\section{InTech Europe}

University Campus STeP Ri

Slavka Krautzeka 83/A

51000 Rijeka, Croatia

Phone: +385 (51) 770447

Fax: +385 (51) 686166

www.intechopen.com

\section{InTech China}

Unit 405, Office Block, Hotel Equatorial Shanghai

No.65, Yan An Road (West), Shanghai, 200040, China

中国上海市延安西路65号上海国际贵都大饭店办公楼 405 单元

Phone: +86-21-62489820

Fax: $+86-21-62489821$ 
(C) 2012 The Author(s). Licensee IntechOpen. This is an open access article distributed under the terms of the Creative Commons Attribution 3.0 License, which permits unrestricted use, distribution, and reproduction in any medium, provided the original work is properly cited. 